\title{
Influence of ambient air on NMR-based metabolomics of exhaled breath condensates
}

\section{To the Editors:}

Nuclear magnetic resonance (NMR)-based metabolomic analysis of exhaled breath condensate (EBC) is a noninvasive approach that has been used to study diseases of the respiratory system $[1,2]$. In a previous study, we questioned the accuracy of NMR-based EBC analysis, demonstrating that the cleaning protocols for reusable condenser parts produce a confounding metabolite fingerprint largely unrelated to the endogenous metabolites from the airways and alveolar fractions of exhaled breath [3]. Here we present new data that provide a qualitative assessment of exogenous contaminants in NMR spectroscopy analysis of EBC, some proceeding from room air. Given the presence of these contaminants in the NMR spectrum, classification of these components as biomarkers of lung function should be regarded with extreme caution. Previous studies have reported the presence of room air components in mass spectrometry analysis of volatiles in exhaled human breath $[4,5]$ and non-volatiles in exhaled human breath condensate [6]. However, although a previous NMR-based metabolomic study of EBC reported the presence of external impurities such as disinfectants or salivary signals [1], the influence of contaminating metabolites in room air was not considered.

In order to analyse the influence of exogenous signals on EBC analysis, room air condensate and EBC from a healthy volunteer were collected with an EcoScreen condenser (Jaeger, Würzburg, Germany). The samples of room air condensate were acquired with a device that continuously pumps room air into the condenser at a flow rate $\left(15 \mathrm{~L} \cdot \mathrm{min}^{-1}\right)$ and tidal volume $(500 \mathrm{~mL})$ typical of normal human breathing. The typical time required for sample acquisition was $15 \mathrm{~min}$. Another set of room air samples was collected by locating a trap for air-contained water-soluble organic compounds between the pump and the condenser to study the origin of the exogenous signals in order to distinguish room air signals from other contaminants. In this case, the ambient air was directed through a chamber containing distilled water so that any water soluble environmental compound will remain in the water. This filtered air was then condensed in the same EcoScreen device used for the whole set of experiments. The reusable parts of the EcoScreen condenser were soaked with hypochlorite solution (15 $\mathrm{min}$ ) and then flushed with deionised distilled water $(15 \mathrm{~min})$. Since hypochlorite solution does not produce ${ }^{1} \mathrm{H}$ NMR signals, this cleaning procedure should avoid disinfectant contamination of the room air condensate and EBC samples.

A gentle stream of nitrogen was passed over the condensates to remove volatile substances. After $5 \mathrm{~min}$ of nitrogen exposure, samples were immediately frozen at $-80^{\circ} \mathrm{C}$ and stored until NMR analysis. In order to evaluate the influence of drying on NMR metabolomic analysis, half of the samples were lyophilised in a Cryodos-50 lyophiliser (Telstar, Barcelona, Spain) before NMR analysis. Non-lyophilised samples were defrosted and $400 \mu \mathrm{L}$ of condensate was transferred to $2 \mathrm{~mL}$ eppendorf tubes. $50 \mu \mathrm{L}$ of $1 \mathrm{mM}$ trimethylsilyl propionate (TSP) in deuterium water $\left(\mathrm{D}_{2} \mathrm{O}\right)$ was added to provide a field frequency lock and internal chemical shift reference. Lyophilised samples were reconstituted with $150 \mu \mathrm{L} \mathrm{D} \mathrm{D}_{2} \mathrm{O}$ and $25 \mu \mathrm{L} 1 \mathrm{mM}$ TSP in $\mathrm{D}_{2} \mathrm{O}$. Both sample sets were transferred to $3 \mathrm{~mm}$ NMR tubes. ${ }^{1} \mathrm{H}-\mathrm{NMR}$ spectroscopy was performed at $700.13 \mathrm{MHz}$ using a BrukerAvance III spectrometer (Bruker Biospin, Rheinstetten, Germany) equipped with TCI CryoProbe, and operating at $4^{\circ} \mathrm{C}$ to reduce metabolite degradation. Standard solvent-suppressed spectra were grouped into 32,000 data points, averaged over 256 acquisitions. Data acquisition lasted $16 \mathrm{~min}$, using a sequence based on the first increment of the Nuclear Overhauser effect spectroscopy (NOESY) pulse sequence to suppress water resonance and limit the effect of B0 and B1 inhomogeneities on the spectra.

The ${ }^{1} \mathrm{H}$ NMR spectra of lyophilised exhaled breath condensates of a healthy subject and from room air show a high correspondence (fig. 1). The same correspondence is observed between the ${ }^{1} \mathrm{H}$ NMR spectral signals of non-lyophilised samples obtained from EBC (fig. S1a) and room air condensates (fig. S1b). Comparison of lyophilised and non-lyophilised samples shows that non-lyophilised spectra present fewer NMR signals, indicating that sample concentration (lyophilisation and resuspension) improves NMR sensitivity. ${ }^{1} \mathrm{H}$ NMR spectra of the lyophilised room air condensate samples clearly show the presence, among other components, of propylene glycol, propionate, acetate, lactate, glycerol, benzoate and formate (fig. S2). Resonances were assigned according to the Human Metabolome Database [7]. Comparison of non-filtered and filtered room air shows that most of the spectral air signals are removed by the collector trap (fig. 2). Nevertheless, filtered room air samples still give ${ }^{1} \mathrm{H}$ NMR signals, including lactate and a non-identified signal (fig. 2b).

Principal components analysis (PCA) [8] was used to analyse the variability between room air condensate samples. The PCA model was developed using eight room air condensates collected on two consecutive days: day 1 , morning $(n=2)$; day 1 , afternoon $(n=2)$; day 2 , morning $(n=2)$; and day 2 , afternoon $(n=2)$. Before PCA, ${ }^{1} \mathrm{H}-\mathrm{NMR}$ spectroscopic data were automatically reduced to integral segments or buckets of equal length $(\delta 0.01 \mathrm{ppm})$ in order to compensate for variations in resonance positions [9] and normalised to the TSP peak of 
a)

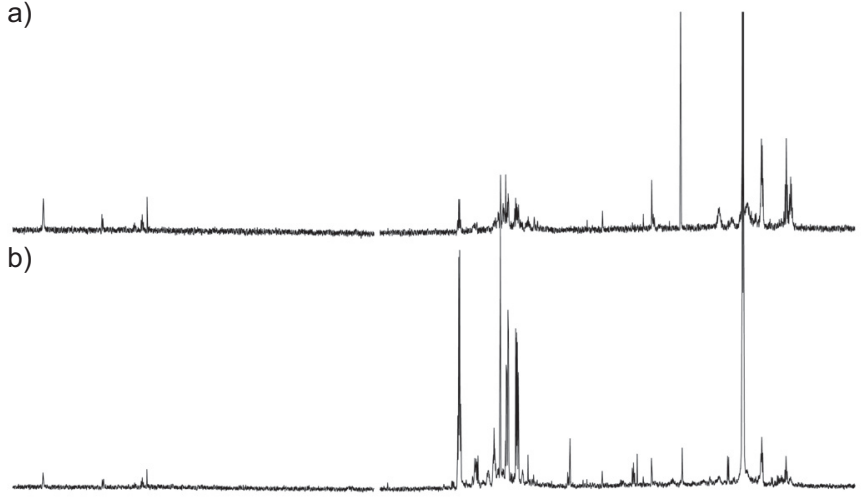

FIGURE 1. ${ }^{1} \mathrm{H}$-nuclear magnetic resonance (NMR) spectra of lyophilised condensates from a) the exhaled breath of a healthy subject and b) room air pumped into the condenser by a continuous air pump. ppm: parts per million.

known concentration. Finally, the data were centred and Pareto scaled. The PCA score plot (fig. S3) shows perfect discrimination along the first principal component between the samples collected on day 1 and day 2 . In addition, a clear separation is also observed along the second principal component between the morning samples and afternoon samples. Furthermore, to evaluate the overfitting of the EBC spectral data, the eight room air condensates were classified into two arbitrary groups (A and B), each containing one sample from each collection time. Partial least squares (PLS) analysis [10] was applied to investigate significant differences between the two groups. Spectral variables were compared by paired t-test. Differences were considered statistically significant at a p-value below 0.05 . Statistical computing and spectral processing were conducted with the Metabonomic package (rel.3.3.1) [11].

In our opinion, the present study demonstrates the presence of an artificial metabolic fingerprint in EBC samples that is unrelated to the endogenous lung metabolism. Comparison of the ${ }^{1} \mathrm{H}$ NMR spectra of EBC samples from a healthy subject with simultaneously collected samples of room air condensates shows an unambiguous correlation. Our ${ }^{1} \mathrm{H}$ NMR analysis demonstrates that room air contains metabolically relevant signals, such as propionate, acetate and lactate, which were proposed in earlier studies to be biomarkers of respiratory diseases [1]. The unsupervised statistical analysis of room air variability in our study shows that the room air metabolite profile correlates with the day and time-of-day of collection. In addition, as proof of statistical pitfalls in metabolomic analysis, the room air condensates were randomly assigned to two arbitrary groups and a supervised PLS analysis was performed to detect differences between these groups. The PLS score plot (fig. S4) shows a clear separation between the two groups, and the PLS model yields a $R^{2}$ value of 98.57 , which would usually be considered to demonstrate a good classification model. Furthermore, 96 spectral regions presented a p-value below 0.05 . The result of this test strengthens the idea that a PLS scores plot alone is insufficient for evaluating differences between

a)

b)

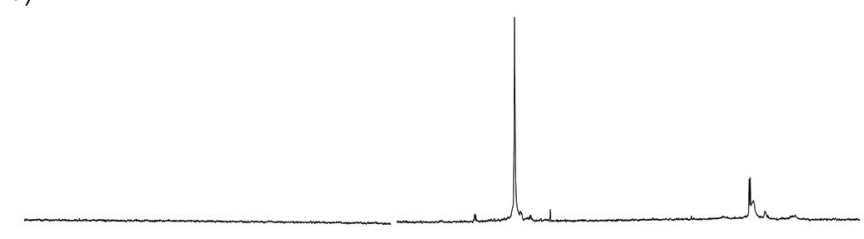

$\begin{array}{llllllllllllllllll}9.0 & 8.5 & 8.0 & 7.5 & 7.0 & 6.5 & 6.0 & 5.5 & 4.5 & 4.0 & 3.5 & 3.0 & 2.5 & 2.0 & 1.5 & 1.0 & 0.5\end{array}$

$1 \mathrm{H}$ NMR chemical shift ppm

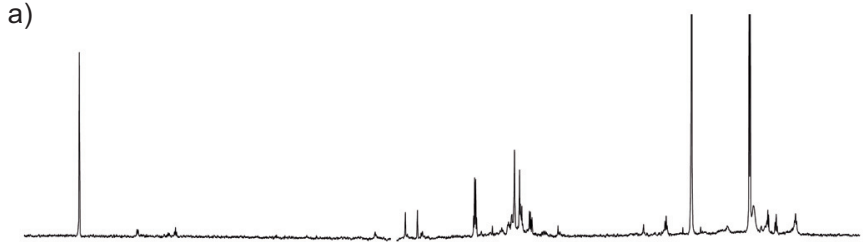

FIGURE 2. ${ }^{1} \mathrm{H}$-nuclear magnetic resonance (NMR) spectra of a) lyophilised condensates from room air and b) room air filtered through a water-soluble organic trap. ppm: parts per million.

groups. The PLS algorithm is fully able to search and identify any difference between metabolic profiles in a multi- and megavariate space in fewer than 50 samples. Therefore, if used without supervision, the PLS algorithm can detect false differences between arbitrary classes. Classification problems in metabolomic data analysis have been widely discussed $[12,13]$, and most models suffer from overfitting. From the results presented here, we can conclude that a simple successful classification of the training data cannot be considered a robust parameter of the model. More realistic information on data predictability and improvements to the quality of the interpretations will require additional datasets for external validation analysis.

The present study demonstrates the presence of an exogenous metabolic profile in the ${ }^{1} \mathrm{H}$ NMR spectra of EBC, reflecting the presence of room air contaminants. Furthermore, we have identified the air room contaminants and demonstrated the variability of the room air condensate spectra by date and timeof-day of experiment. Room air condensates contain the same metabolites postulated as disease biomarkers in previous publications [1], and our present analysis of PLS tests brings into question the statistical robustness of the models developed for NMR analysis of EBC.

In our opinion, researchers who quantify metabolites in breath must compensate somehow for the analytes presented in background air. This is perhaps a problem for NMR approaches since filtering out these signals during acquisition is not possible. However, this is not a problem for mass spectrometry (MS)-based methods. Thus, we consider that there are essentially three working options. 1) Ignore the problem. This is probably the worst scenario and unfortunately most of the published papers on the topic of EBC make no mention at all to this problem. Our results demonstrate that this is not only a problem for volatile metabolites, but also for metabolites in EBC. Our conclusion is that EBC-based breathomic data are skewed and results may be overfitted if these metabolites remain in the analysis. 2) Supply the subject with medical air before and during 
EBC data collection. At this moment, this approach needs to be validated in a rigorously controlled study and probably the only solution for any relevant NMR based EBC data. In theory, this new control could provide an unambiguous picture of breath metabolism. 3) Subtracting the air background from the breath signal (alveolar gradient). This method is currently applied in MS breath analysis by measuring the metabolic concentration in volatiles (also in EBCs) and subtracting (filtering) the analytes from room air. Details about room air measurement have been previously reported [14]. A similar approach cannot be easily translated to NMR-based metabolomic data.

In summary, we are proposing that medical air inhalation should be a requirement of future NMR-based metabolomic analysis.

Jose L. Izquierdo-García*,\#, Germán Peces-Barba and Jesús Ruiz-Cabello*,\#,+

*CIBERES, CIBER EnfermedadesRespiratorias, ${ }^{\#} \mathrm{CNIC}$, Centro Nacional de Investigaciones Cardiovasculares, "Fundación Jiménez Díaz-CAPIO, and ' Universidad Complutense de Madrid, Dpt Química-Física II, Madrid, Spain.

Correspondence: Jesús Ruiz-Cabello, Centro Nacional de Investigaciones Cardiovasculares, Melchor Fernández Almagro 3, Madrid 28029, Spain. E-mail: ruizcabe@cnic.es

Support Statement: This research was supported by the PINET European Network (ITN-FP7-264864) and the Spanish Ministry of Economy and Competition (SAF2011-25445). The CNIC is supported by the Pro-CNIC Foundation and the Ministry of Economy and Competition.

Statement of Interest: None declared.

Acknowledgements: We thank D. Molero of the NMR Center at the Complutense University of Madrid, Madrid, Spain for NMR spectra acquisition. S. Bartlett (CNIC, Madrid, Spain) provided English editing.

\section{REFERENCES}

1 de Laurentiis G, Paris D, Melck D, et al. Metabonomic analysis of exhaled breath condensate in adults by nuclear magnetic resonance spectroscopy. Eur Respir J 2008; 32: 1175-1183.

2 Carraro S, Rezzi S, Reniero F, et al. Metabolomics applied to exhaled breath condensate in childhood asthma. Am J Respir Crit Care Med 2007; 175: 986-990.

3 Izquierdo-Garcia JL, Peces-Barba G, Heili S, et al. Is NMR-based metabolomic analysis of exhaled breath condensate accurate? Eur Respir J 2011; 37: 468-470.

4 Pleil JD. Role of exhaled breath biomarkers in environmental health science. J Toxicol Env Health B Crit Rev 2008; 11: 613-629.

5 Mazzone PJ. Analysis of volatile organic compounds in the exhaled breath for the diagnosis of lung cancer. J Thorac Oncol 2008; 3: 774-780.

6 Kurova V, Kononikhin A, Sakharov D, et al. Exogenous proteins in exhaled human breath condensate. Russian J Bioorg Chem 2010; 37: 48-52.

7 Wishart DS, Tzur D, Knox C, et al. HMDB: the Human Metabolome Database. Nucleic Acids Res 2007; 35: D521-D526.

8 Hotelling H. Analysis of a complex of statistical variables into principal components. J Edu Psychol 1933; 24: 417-441.

9 Holmes E, Foxall PJD, Nicholson JK, et al. Automatic data reduction and pattern recognition methods for analysis of ${ }^{1} \mathrm{H}$ nuclear magnetic resonance spectra of human urine from normal and pathological states. Anal Biochem 1994; 220: 284-296.

10 Kramer R. Chemometric Techniques for Quantitative Analysis. New York, Marcel Dekker, 1998.

11 Izquierdo-Garcia J, Rodriguez I, Kyriazis A, et al. A novel Rpackage graphic user interface for the analysis of metabonomic profiles. BMC Bioinformatics 2009; 10: 363.

12 Westerhuis J, Hoefsloot H, Smit S, et al. Assessment of PLSDA cross validation. Metabolomics 2008; 4: 81-89.

13 Westerhuis J, van Velzen E, Hoefsloot $\mathrm{H}$, et al. Discriminant $\mathrm{Q}^{2}$ $\left(\mathrm{DQ}^{2}\right)$ for improved discrimination in PLSDA models. Metabolomics 2008; 4: 293-296.

14 Martin A, Farquar G, Jones A, et al. Human breath analysis: methods for sample collection and reduction of localized background effects. Anal Bioanal Chem 2010; 396: 739-750.

\section{Extracorporeal membrane oxygenation in a nonintubated patient with acute respiratory distress syndrome}

\section{To the Editors:}

Endotracheal intubation and mechanical ventilation are mainstays in the management of patients with acute respiratory distress syndrome (ARDS), but this treatment strategy exposes the patient to several risks and complications. A small number of ARDS patients can be treated with noninvasive ventilation and these patients have less ventilator-associated pneumonia and a lower mortality rate [1]. However, failure to improve oxygenation with noninvasive ventilation indicates the need for endotracheal intubation [1].

In patients with severe respiratory failure, extracorporeal membrane oxygenation (ECMO) is increasingly being used on top of mechanical ventilation to facilitate oxygenation and protective ventilation [2]. A novel concept is the use of ECMO in awake, spontaneously breathing patients to avoid the complications of invasive ventilation. So far, "awake ECMO" 